\title{
Estruturação do PBL para Aplicação em Disciplinas do Curso de Graduação em Administração
}

\author{
Roberto Portes Ribeiroํㅗ Adriana Backx Noronha Viana² \\ ${ }^{1}$ Universidade Federal de Santa Maria \\ ¿Universidade de São Paulo
}

* Autores para correspondência: robertor@ufsm.br; backx@usp.br

\begin{abstract}
RESUMO
As dificuldades encontradas no processo de ensino-aprendizagem de disciplinas do curso de graduação em Administração motivam a busca por iniciativas capazes de proporcionar um ambiente de aprendizagem flexível que integre teoria e prática empresarial, conforme propõem as Diretrizes Curriculares do curso de Administração. Esta pesquisa tem por objetivo identificar as definições conceituais e estabelecer os procedimentos operacionais para subsidiar a estruturação do PBL (Aprendizagem Baseada em Problemas) no processo de ensino-aprendizagem de Administração. $\mathrm{O}$ método de pesquisa com ênfase em uma abordagem qualitativa de caráter descritivo envolveu levantamento bibliográfico, entrevista e observação, a fim de identificar definições conceituais que subsidiem a estruturação do PBL para aplicação em disciplinas do curso de graduação em Administração. Dadas as limitações em aplicar o PBL a uma disciplina, considera-se que essa iniciativa gerou resultados no sentido do entendimento e da estruturação da aplicação do método no contexto proposto, de maneira a fornecer bases para futuros estudos e estruturação do curso de Administração utilizando-se integralmente do método.
\end{abstract}

Palavras-Chave: PBL; Curso de Administração; Ensino.

\begin{abstract}
The difficulties encountered in the teaching-learning undergraduate Administration course subjects motivate the search for initiatives to provide a flexible learning environment that integrates theory and business practice as propose the Administration Course Curriculum Guidelines. This research aims to identify the conceptual definitions and establish the operational procedures to support the structuring of the PBL (Problem-Based Learning) in the teaching-learning process management. The research method with emphasis on a qualitative approach involved descriptive literature, interview and observation to identify conceptual definitions that support the structure of PBL for application in the Administration undergraduate course subjects. Given the limitations of applying PBL to a subject, it is considered that this initiative generated results in the sense of understanding and structuring of the method the proposed context, providing a basis for further studies and structuring of the Administration undergraduate courses using full PBL.
\end{abstract}

Keywords: PBL; Administration Course; Teaching.

\section{Introdução}

As diretrizes curriculares devem ser observadas como guia de orientação para que os cursos de graduação em Administração atendam a crescente heterogeneidade da formação e as tendências contemporâneas, a fim de que se possam inserir no mercado profissionais com competências, habilidades e atitudes condizentes com a realidade de mercado em nível de graduação, como uma etapa inicial da formação continuada. Sendo assim, os gestores envolvidos com cursos de graduação em
Administração precisam estar conscientes de que o desenvolvimento da capacidade de aprender a aprender deve ser respaldado por práticas pedagógicas que permitam ao estudante desenvolver seu potencial criativo e análise crítica em relação ao que ocorre nas organizações, visando ao exercício da cidadania (ANDRADE \& AMBONI, 2004).

No Brasil, apesar da necessidade de incorporar novas técnicas e habilidades aos profissionais que atualmente são solicitados pelo mercado, restrições e limitações ainda persistem na maioria dos cursos 
de Administração, tais como: o papel acomodado de repassador do conhecimento do professor, a passividade dos estudantes e as instituições que não oferecem estrutura e apoio necessários para a mudança do sistema educacional (LIMA \& SILVA, 2013). Para atender a essa demanda, novos métodos de ensino-aprendizagem como, por exemplo, o PBL (Aprendizagem Baseada em Problemas) poderiam ser utilizados para contribuir na formação de profissionais com capacidade de adaptação e atualização ao mercado de trabalho, além de fomentar o interesse dos estudantes e seu envolvimento para a formação plena como cidadãos.

Mesmo com as recentes iniciativas de Souza e Verdinelli (2014) e Borochovicius e Tortella (2014) de estudar a utilização do PBL em disciplinas nos cursos de graduação em Administração, seu uso ainda é incipiente frente a outros cursos, tais como: Medicina, Engenharia e Enfermagem (SHERWOOD, 2004; ZABIT, 2010).

Diante desse contexto, este estudo objetiva identificar as definições conceituais e estabelecer os procedimentos operacionais para subsidiar a estruturação do PBL no processo de ensino-aprendizagem de Administração, em cinco partes: primeiramente se desenvolve a introdução, na qual se esclarecem a relevância do tema e os objetivos. A segunda seção contém a fundamentação teórica a respeito da questão do ensino-aprendizagem, enfatizando o PBL no processo de ensino-aprendizagem de Administração. A terceira parte aborda o método utilizado na pesquisa. $\mathrm{O}$ quarto tópico apresenta os resultados encontrados no estudo, e a quinta parte traz as considerações finais.

\section{Referencial Teórico}

\section{Métodos de Ensino-Aprendizagem}

Rangel (2013) abordou os métodos de ensino como meios de dinamização das aulas e a relação prática-teoria como condição indispensável para tornar o conhecimento acessível à compreensão e, portanto, à elaboração e à (re)construção pelo estudante, tendo em vista, sobretudo, a articulação efetiva entre ensino e aprendizagem. Marion e 40
Marion (2006) apontaram a tendência de criticar o estilo de ensino tradicional baseado nas preleções dos professores como fonte primária do ensino, pois, nesse processo em que o estudante é agente passivo de aprendizagem, faz-se necessário memorizar regras, definições e procedimentos sem se entender o porquê de tais procedimentos. A partir disso, os referidos autores analisaram treze métodos de ensino possíveis de aplicação às disciplinas na área de Administração e constataram seus benefícios e dificuldades.

A aula expositiva é apenas um dos métodos de ensino que podem ser utilizados nas salas de aula dos cursos de Administração. É possível escolher outros métodos que favoreçam uma participação ativa do estudante e adotem procedimentos de coparticipação do professor como orientador de estudos, alternando sua orientação com trabalhos independentes dos estudantes e articulando teoria e prática. Dentre esses métodos, destaca-se o PBL.

\section{$P B L$}

Barrows (1996), um dos pioneiros no desenvolvimento e pesquisa do PBL, sustentou que o método promove o desenvolvimento do pensamento crítico, de estratégias de aprendizagem independente, de resolução de problemas, de habilidades sociais e de comunicação, pois a aprendizagem ocorre por intermédio de problemas reais ou simulados que desafiam e estimulam a aquisição e o compartilhamento de conhecimentos.

O PBL é um método baseado no princípio do uso de problemas como ponto de partida para incentivar a aquisição e a integração de novos conhecimentos. Barrows (1996) destacou que a colocação de desafios na forma de problemas relevantes à futura atuação dos estudantes antes da apresentação da teoria é considerada como o núcleo fundamental do método. Hmelo-Silver (2004) conceituou o PBL como o método de ensino em que os estudantes aprendem através da resolução de problemas e que foca em problemas complexos que não têm uma única resposta correta. Na literatura, o PBL ainda está fortemente relacionado ao ensino de Medicina, por integrar 
conceitos e teorias às práticas médicas, promovendo o domínio dos conhecimentos dessa área, bem como o desenvolvimento de habilidades e atitudes, necessárias ao profissional da saúde.

Três autores foram fundamentais no processo de estruturação da aplicação do PBL. Inicialmente, Hmelo-Silver (2004) apresentou um ciclo entendido como processo tutorial PBL e destacou a importância da identificação de conhecimentos deficientes relevantes para resolver o problema, como uma importante parte desse processo. Depois Savery (2006) relacionou uma lista de práticas consideradas características da filosofia, estratégias e táticas do PBL, além de métodos utilizados e as competências específicas desenvolvidas. Por fim, Ribeiro (2008) apresentou os fundamentos e elementos principais do PBL, descrevendo uma sequência de ciclos de trabalho com problemas.

Além da estruturação, a adequação do PBL para a utilização em disciplinas ligadas aos cursos de Administração deve considerar a necessidade de suportes educacionais para apoiar o desenvolvimento de habilidades de resolução de problemas (KIILI, 2007), o papel de mediação do tutor no processo de ensino e aprendizagem (SPRONKENSMITH \& HARLAND, 2009) e os problemas que permitam aos estudantes aprender por meio da aplicação e da adaptação dos conceitos teóricos às situações práticas (AMBROSINI, BOWMAN \& COLLIER, 2010).

As vantagens e desvantagens da aplicação do PBL no contexto brasileiro foram discutidas por Escrivão Filho e Ribeiro (2009), e ainda Borochovicius e Tortella (2014) e Souza e Verdinelli (2014) realizaram avaliações do PBL utilizado nas disciplinas de cursos de Administração. Desse modo, a opção pelo PBL propicia o ensino com uma visão complexa que proporciona aos estudantes a convivência com a diversidade de opiniões, convertendo as atividades do método em situações ricas e significativas para a produção do conhecimento e a aprendizagem para a vida. Propicia o acesso a maneiras diferenciadas de aprender e, especialmente, de aprender a aprender.

\section{Método}

As etapas e os procedimentos deste estudo, concebidos de acordo com as indicações de Gil (2010), foram conduzidos em duas partes: preparação do método $\mathrm{PBL}$ e estruturação da utilização do PBL.

A primeira etapa de preparação do PBL envolveu três procedimentos: inicialmente, a revisão da literatura; em seguida, o desenvolvimento de uma entrevista com um profissional que foi formado pelo método PBL na graduação em Medicina; e, por fim, o acompanhamento de uma disciplina ministrada por meio do PBL na graduação em Engenharia Civil.

O intuito da revisão de literatura foi proporcionar um panorama do estágio do conhecimento e das principais questões sobre o tema PBL, relacionando-o com outros métodos de ensino-aprendizagem em Administração.

O segundo procedimento desta etapa envolveu o desenvolvimento de uma entrevista a respeito da utilização do PBL no curso de graduação em Medicina: foi concedida por um médico formado pela Faculdade de Medicina de Marília-SP, na qual o curso de graduação em Medicina foi pioneiro no Brasil ao implementar e funcionar exclusivamente pelo método PBL.

Essa entrevista caracteriza-se por ser uma amostra classificada por Sampieri, et. al (2013) de caso típico, cujo objetivo é a profundidade e a qualidade da informação, e não a quantidade nem a padronização. $\mathrm{O}$ roteiro de entrevista semiestruturada considerou aspectos práticos, éticos e teóricos de acordo com as indicações dos referidos autores, e foi concebido em dez partes constituídas de perguntas abertas, começando pelas mais gerais até as mais específicas e com a intenção de que o participante compartilhe sua perspectiva e experiência sobre o fenômeno.

O terceiro procedimento envolveu o acompanhamento por observação qualitativa da aplicação do PBL em uma disciplina de Administração e Empreendedorismo no curso de graduação em Engenharia Givil da Universidade de São Paulo na cidade de São Carlos, sob supervisão do Prof. 
Edmundo Escrivão Filho, especialista na aplicação do PBL em disciplinas.

Conforme Sampieri, et. al (2013), essa amostra pode ser classificada como amostra de especialista, adotada quando se precisa da opinião de indivíduos especialistas em determinado tema. Nessa observação qualitativa, foram realizadas anotações descritivas e interpretativas. A disciplina foi planejada para operar com quatro módulos, sendo observado o segundo módulo, que ocorreu em três aulas consecutivas de duração de uma hora e cinquenta minutos. $\mathrm{O}$ ambiente virtual da disciplina foi disponibilizado para acesso e serviu de preparação para as aulas.

Os procedimentos adotados na etapa anterior serviram para subsidiar a análise nessa etapa e possibilitar a construção de mapas conceituais para estruturar o processo de utilização do PBL em três fases. Os mapas conceituais foram construídos com o auxílio do software CmapTools, criado e disponibilizado gratuitamente pelo Institute for Human and Machine Cognition.

Estruturação do Processo de Utilização do PBL em uma Disciplina do Curso de Graduação em Administração

O resultado do entendimento e da sistematização dos conceitos e procedimentos do PBL foi a estruturação do processo de utilização do método em três etapas: contexto pedagógico, problemas e etapas de aplicação, que podem ser observadas na Figura 1. A construção dos mapas conceituais considerou a forma hierárquica clássica de representação dos eventos na ordem em que ocorrem através do tempo, dividindo-os em três mapas que representam as fases de utilização do PBL.

\section{Contexto Pedagógico}

Savery (2006) descreveu os princípios do PBL, que envolvem a capacidade de pensar criticamente, analisar e resolver os problemas da realidade, para localizar, avaliar e usar de forma adequada os recursos de aprendizagem, para trabalhar cooperativamente, para demonstrar habilidades de comunicação eficaz, e de utilizar o conhecimento e habilidades intelectuais. O PBL é um método de ensino-aprendizagem pautado na construção do conhecimento pelo estudante, com orientação do professor, como um facilitador da aprendizagem, e a responsabilidade do aprendiz de ser autodirecionado e autorregulado é um ponto-chave. Sendo assim, o primeiro passo para a implementação do método é mapear e incorporar seus princípios norteadores.

A próxima etapa de análise do contexto pedagógico abrange a formação de um grupo de planejamento do curso que envolve docentes de todas as áreas que, com a pluralidade de pontos de vista, examinarão o curso como um todo, (re) analisando e (re)estruturando sua visão e missão, (re)alinhando a estrutura curricular com as tendências do sistema educacional, (re)examinando o perfil do egresso de acordo com as Diretrizes Curriculares do curso, de modo a desenvolver um

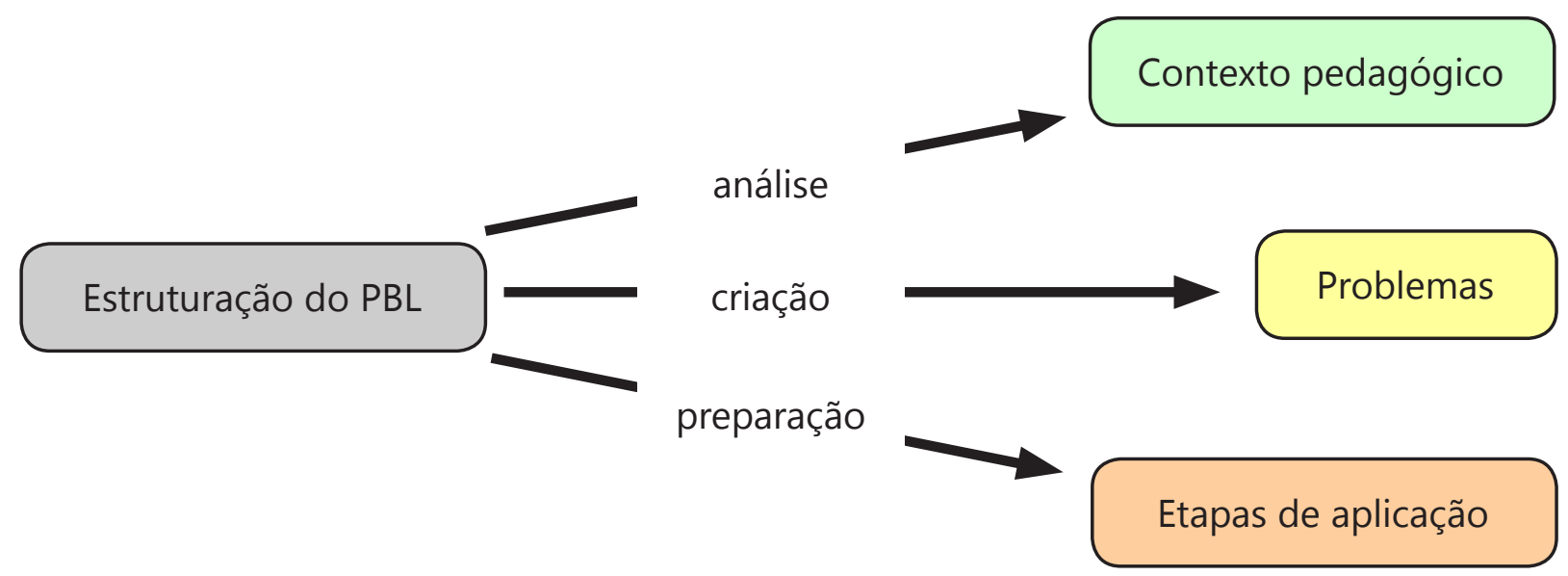

Figura 1 - Estruturação do processo de utilização do PBL. Fonte: Autores. 
projeto formativo contextualizado que contemple estudos teórico-práticos relacionados à ação profissional.

O grupo de planejamento do curso deve ser desdobrado em oficinas de trabalho que, de acordo com a estrutura curricular, definem o público-alvo e os conteúdos dos módulos ou unidades educacionais. Dado que a abordagem dos problemas deve ser realizada de forma interdisciplinar para garantir os conhecimentos científicos necessários, associados a uma visão humanista e ética da profissão, segue-se a construção de árvores temáticas, estruturadas a partir de temas abrangentes até temas específicos, como os abordados em planos de ensino de disciplinas; assim o objetivo, o conteúdo, a estratégia de ensino, o processo de avaliação, o cronograma e a bibliografia devem ser transformados.

Conforme Roesch e Fernandes (2007), os objetivos educacionais, classificados em domínios cognitivo, psicomotor e afetivo, servem para definir o nível de aprendizagem que se espera seja atingido pelos estudantes. A Figura 2 explicita o mapa conceitual de análise do contexto pedagógico que inicia com o mapeamento dos princípios do PBL descritos por Savery (2006) e finaliza com a definição dos objetivos educacionais classificados por Roesch e Fernandes (2007).

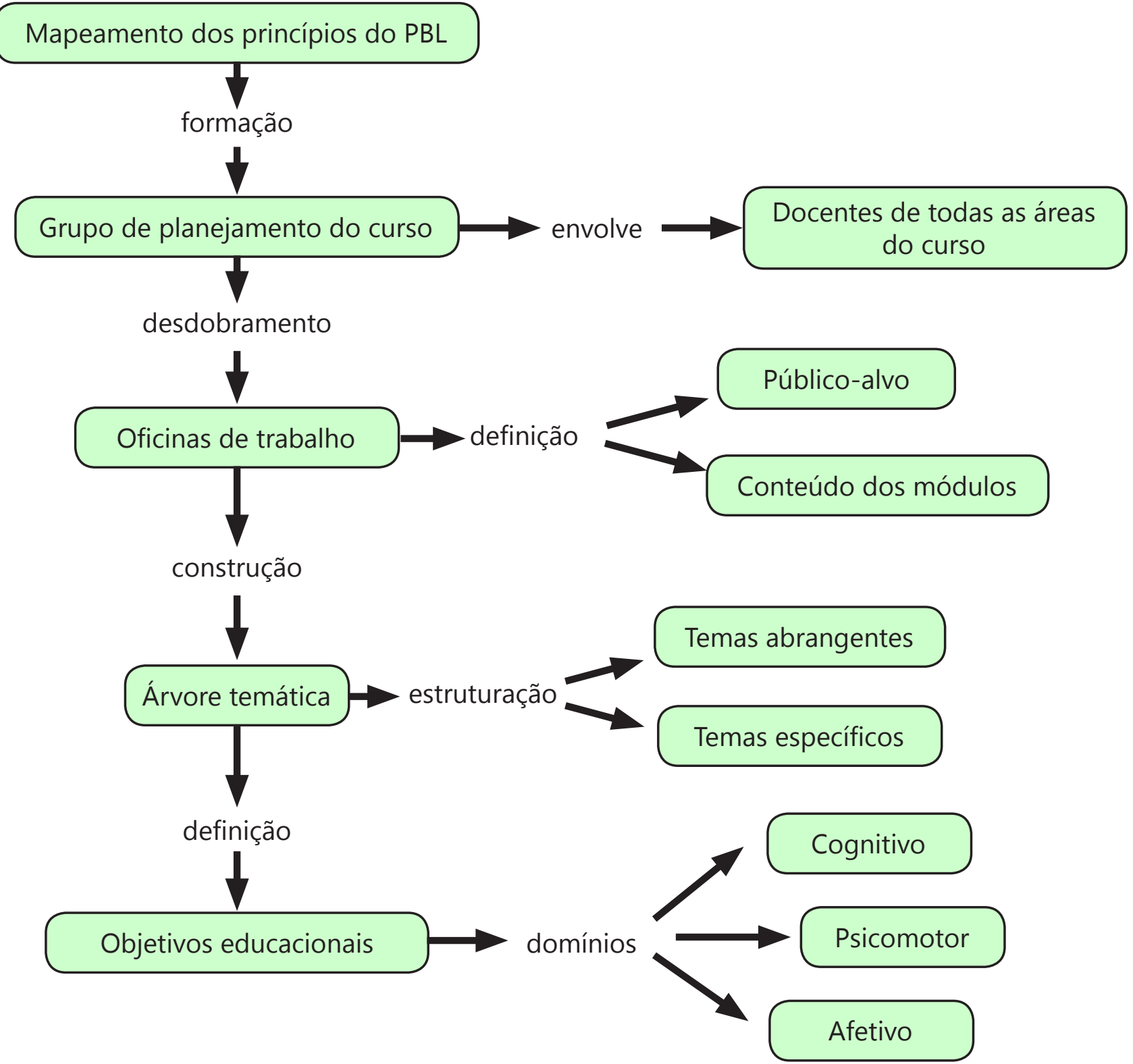

Figura 2 - Mapa conceitual de análise do contexto pedagógico. Fonte: Autores. 


\section{Problemas}

Ambrosini, et. al (2010) ressaltaram a importância dos problemas para que os estudantes aprendam por meio da aplicação e da adaptação dos conceitos teóricos às situações práticas. Abell (1997) enumerou as características essenciais dos problemas para cumprir a função de instrumentos de ensino-aprendizagem. Roesch e Fernandes (2007) sugeriram passos para a construção de problemas relacionados ao ensino-aprendizagem na área de Administração. Segundo os autores, o professor, ao escrever o problema, deve pensar no contexto pedagógico de aplicação em aula.

Segundo Roesch e Fernandes (2007) e Abell (1997), o escritor de problemas de ensino-aprendizagem necessita identicar situações problemáticas que representem os tópicos selecionados, ou seja, leitura de publicações na mídia, histórias contadas por colegas, memórias de sua própria experiência de consultoria ou de trabalho em organizações, bem como de trabalhos acadêmicos. A questão é saber resgatar tais histórias, o que implica colher informações e criar um texto que seja útil para o ensino-aprendizagem, que trate de problemas locais e assim envolva os estudantes na discussão.

Coletar dados, definir a estrutura e narrar são processos que se entrelaçam na construção de um problema. Uma situação-problema é narrada como uma história, e contar uma história implica integrar diversas informações de acordo com uma sequência lógica. A estrutura básica compreende parágrafo inicial, corpo do caso e parágrafo final. Ao contrário de um texto acadêmico, num problema para ensino-aprendizagem não há hipóteses para guiar a coleta de dados. A história vai se formando por intermédio do entrelaçamento entre o contexto e a situação-problema.

A coleta de dados envolve a escolha do tipo de informação mais conveniente de ser utilizada no problema, que pode ser obtida pela condução de entrevistas ou utilização de observação. Apresentação clara, observância das regras gramaticais, precisão vocabular e versatilidade na construção das frases fazem parte da revisão.
No teste, o problema é aplicado em sala de aula, reproduzindo as condições de ensino para as quais foi concebido. O problema pode não gerar debate, e alterações podem vir a ser necessárias até que a versão final esteja pronta.

A Figura 3 expõe o mapa conceitual de criação dos problemas no método $\mathrm{PBL}$, que seguiu basicamente as etapas preconizadas por Roesch e Fernandes (2007) e as características elencadas por Abell (1997).

\section{Etapas da Aplicação do PBL}

As etapas de aplicação do PBL consideraram o ciclo de Hmelo-Silver (2004), entendido como um processo tutorial PBL que destacou a importância da identificação de conhecimentos deficientes relevantes para resolver problemas. Neste ciclo, o professor apresenta aos estudantes o contexto do problema, e a identificação dos fatos deve ocorrer por parte dos estudantes. O trabalho do tutor segue com o auxílio na representação do problema, implicando maior compreensão e gerando hipóteses. Os conhecimentos deficientes, sinônimo de questões de aprendizagem escolhidas pelo tutor na elaboração dos problemas, são pesquisados pelos estudantes, que desenvolvem a autoaprendizagem à medida que pesquisam. Desse modo, eles aplicam os novos conhecimentos e avaliam as hipóteses acerca do que aprenderam. Ao final, concluem cada problema mediante abstração do conhecimento adquirido. Dentre as vantagens desse ciclo, Hmelo-Silver (op. cit) destacou que os estudantes desenvolvem habilidades para solucionar o problema, colaboração e aprendizagem autônoma.

O detalhamento das etapas de aplicação do PBL seguiu a configuração utilizada por Ribeiro (2008), que apresentou o ciclo de trabalho com um problema no PBL. Este ciclo inicia-se com a apresentação de um problema, o qual é analisado e definido pelos estudantes em grupos; em um segundo momento, eles discutem livremente e levantam hipóteses, confrontando-as com os dados do problema, tentando solucioná-lo com conhecimentos prévios. Dado o insucesso na 


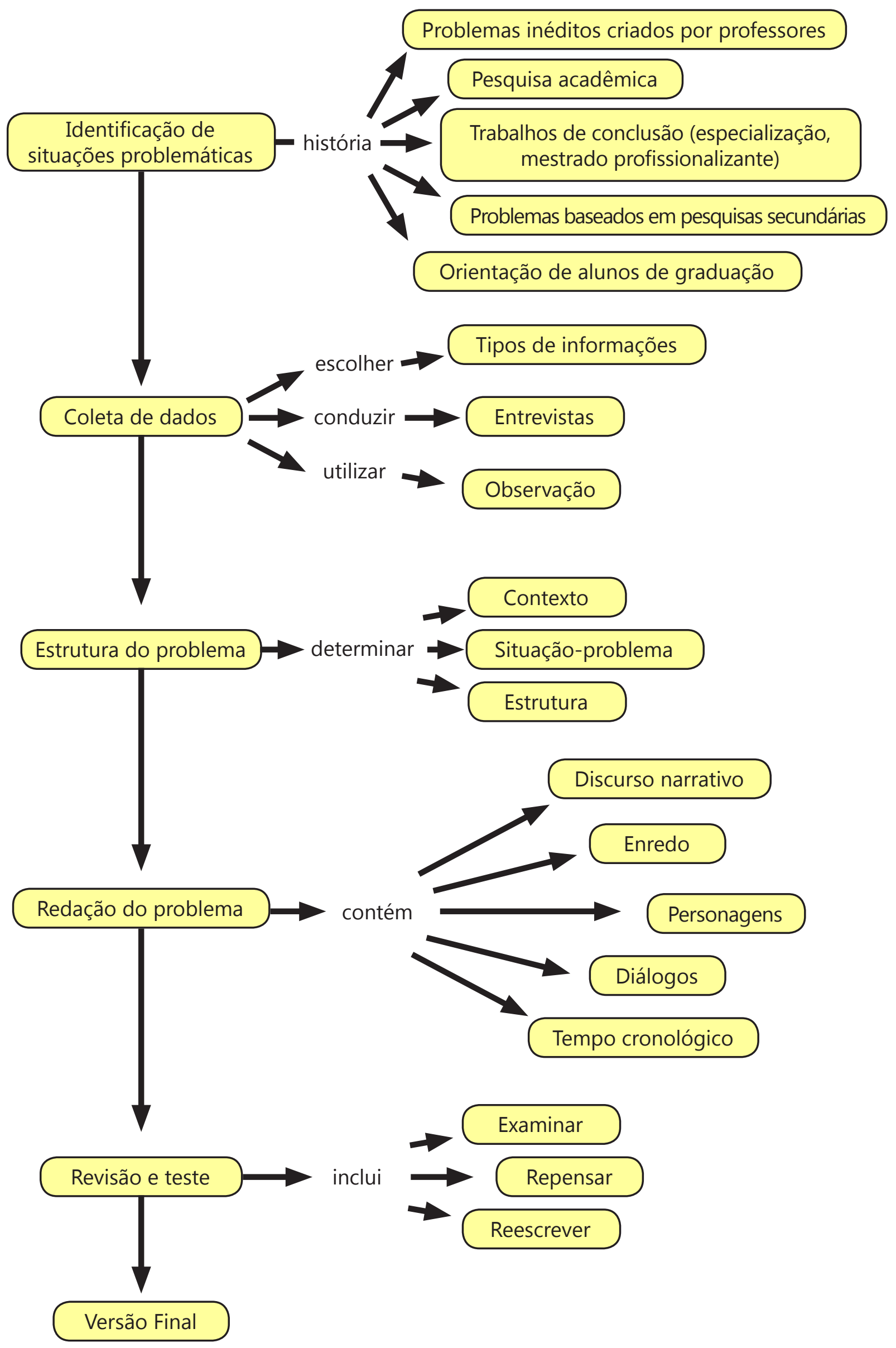

Figura 3 - Mapa conceitual de criação dos problemas. Fonte: Autores. 
solução do problema com os conhecimentos de que dispõem, os estudantes levantam os pontos de aprendizagem necessários para solucioná-lo, cuja importância foi destacada por Hmelo-Silver (2004).

$\mathrm{Na}$ etapa seguinte, os estudantes planejam o trabalho do grupo. Desse modo, buscam os conceitos e informações de forma autônoma, de acordo com seu plano de trabalho coletivo. Eles compartilham informações e conceitos no grupo em encontros não tutorados. E aplicam os conhecimentos desenvolvidos na resolução do problema tantas vezes quanto forem necessárias, até atingirem uma solução.

A etapa seguinte requer a produção de algo concreto apresentado para o tutor e outros grupos durante as sessões tutoriais. Por fim, ocorre o processo de avaliação: os estudantes avaliam o processo, seu produto, o trabalho em grupo, seu próprio desempenho e dos pares. A Figura 4 mostra o fluxograma das etapas de aplicação do PBL que seguiram a configuração utilizada por Ribeiro (2008) e Hmelo-Silver (2004).

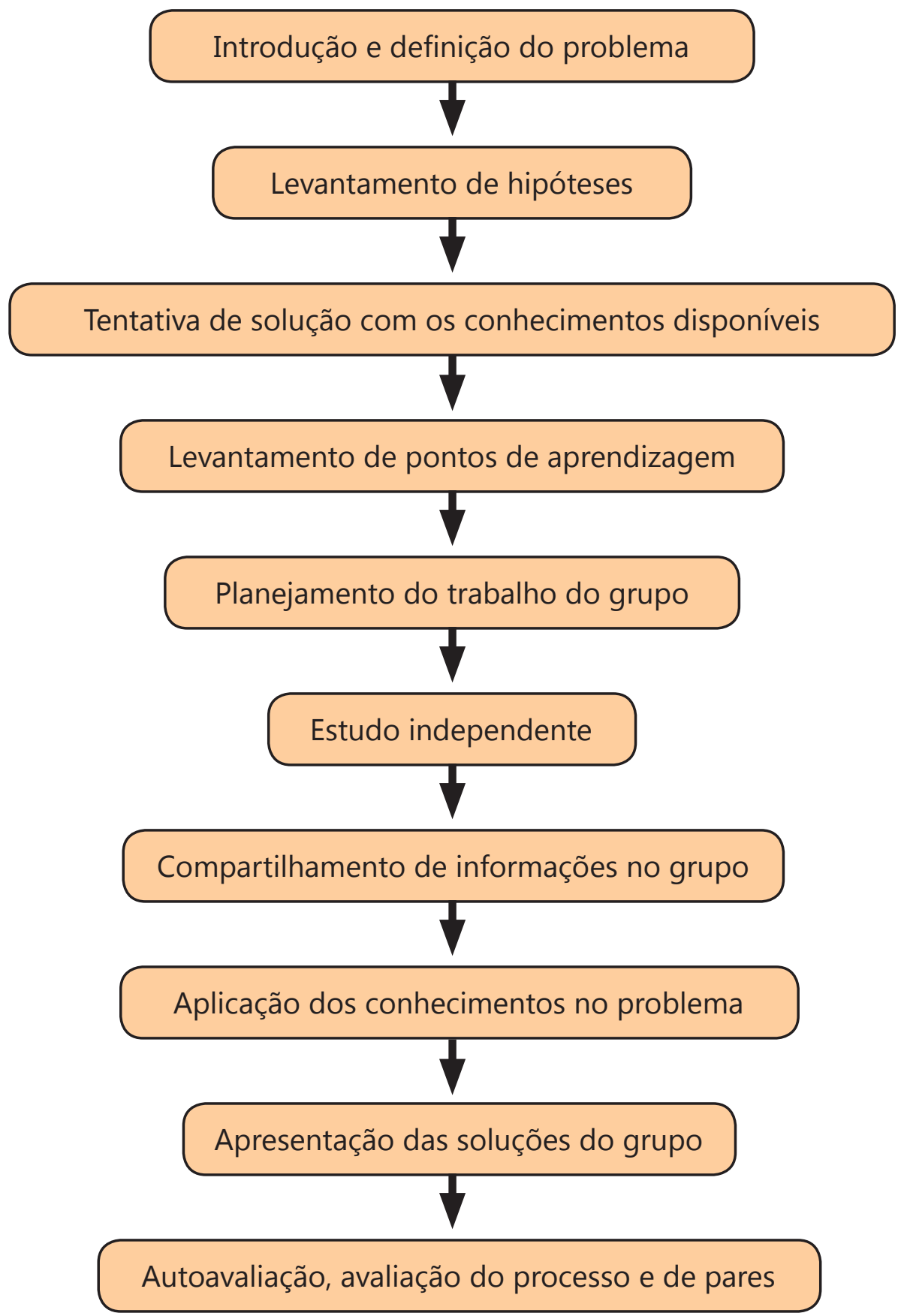

Figura 4 - Etapas de aplicação do PBL. Fonte: Adaptado de Ribeiro (2008, p. 27). 


\section{Considerações Finais}

O PBL foi estruturado com a intenção de fortalecer o processo de ensino-aprendizagem na área de Administração, estimulando a resolução de problemas gerenciais, o que pode indicar possíveis avanços na área de Administração como ocorreu em outras áreas, notadamente na Medicina. De acordo com Savery (2006), o PBL deve ser aplicado considerando um curso como um todo, em que o método deve ser a base de um currículo pedagógico e não parte de um currículo didático, de modo que o aprendizado possa ser integrado através de uma grande variedade de temas e disciplinas. Por outro lado, Escrivão Filho e Ribeiro (2009) aplicaram parcialmente o PBL em uma disciplina de um curso de Engenharia com resultados significativos. Dadas as limitações no contexto de pensar e aplicar o PBL a uma disciplina de graduação, considera-se que essa iniciativa pode gerar resultados no sentido de se entenderem o funcionamento e a aplicação do PBL em disciplinas de graduação em Administração, fornecendo bases para futuros estudos e estruturação do curso utilizando-se integralmente do PBL como a base estrutural do currículo pedagógico.

\section{Agradecimento \\ CNPq.}

\section{Referências Bibliográficas}

ABELL, D. "What Makes a Good Case?". ECCHO - The Newsletter of the European Case Clearing House, vol. 17, n. 1, 1997, pp. 4-7.

AMBROSINI, V.; BOWMAN, C. \& COLLIER, N. "Using Teaching Case Studies for Management Research". Strategic Organization, vol. 8, n. 3, 2010, pp. 206-229.

ANDRADE, R. O. B. \& AMBONI, N. Gestão de Cursos de Administração: Metodologias e Diretrizes Curriculares. São Paulo: Prentice Hall, 2004.

BARROWS, H. S. "Problem-Based Learning in Medicine and beyond: a Brief Overview". In: WILKERSON, L. \& GIJSELAERS, W. H. (eds.). Bringing Problem-Based Learning to Higher Education: Theory and Practice. San Francisco: Jossey-Bass, 1996, pp. 3-12.

BOROCHOVICIUS, E. \& TORTELLA, J. G. B. "Aprendizagem Baseada em Problemas: um Método de Ensino-Aprendizagem e suas Práticas Educativas". Ensaio: Avaliação e Políticas Públicas em Educação, vol. 22, n. 38, 2014, pp. 263-294.
ESCRIVÃO FILHO, E. \& RIBEIRO, L. R. C. "Aprendendo com PBL - Aprendizagem Baseada em Problemas: Relato de uma Experiência em Cursos de Engenharia da EESG-USP". Minerva, vol. 6, n. 1, 2009, pp. 23-30.

GIL, A. C. Como Elaborar Projetos de Pesquisa. 5. ed. São Paulo: Atlas, 2010.

HMELO-SILVER, C. E. "Problem-Based Learning: What and How Do Students Learn?". Educational Psychology Review, vol. 16, n. 3, 2004, pp. 235-266.

KIILI, K. "Foundation for Problem-Based Gaming". British Journal of Educational Technology, vol. 38, n. 3, 2007, pp. 394-404.

LIMA, T. B. \& SILVA, A. B. "Difusão das Perspectivas Teóricas da Aprendizagem na Formação de Administradores". Revista Iberoamericana sobre Calidad, Eficacia y Cambio en Educación, vol. 11, n. 3, 2013, pp. 5-30.

MARION, J. C.; MARION, A. L. C. Metodologias de Ensino na Área de Negócios: para Cursos de Administração, Gestão, Contabilidade e MBA. São Paulo: Atlas, 2006.

RANGEL, M. Métodos de Ensino para a Aprendizageme a Dinamização das Aulas. 6 ed. Campinas: Papirus, 2013.

RIBEIRO, L. R. C. "Aprendizagem Baseada em Problemas (PBL) na Educação em Engenharia". Revista de Ensino de Engenharia, vol. 27, n. 2, 2008, pp. 23-32.

ROESCH, S. M. A. \& FERNANDES, F. Como Escrever Casos para o Ensino de Administração. São Paulo: Atlas, 2007.

SAMPIERI, R. H.; COLLADO, G. F. \& LUCIO, M. P. B. Metodologia de Pesquisa. 5 ed. Porto Alegre: Penso, 2013.

SAVERY, J. R. "Overview of Problem-Based Learning: Definitions and Distinctions". The Interdisciplinary Journal of Problem-based Learning, vol. 1, $\mathrm{n}$. 1, 2006, pp. 9-20.

SHERWOOD, A. L. "Problem-Based Learning in Management Education: a Framework for Designing Context". Journal of Management Education, vol. 28, n. 5, 2004, pp. 536-557.

SOUZA, N. R. \& VERDINELLI, M. A. "Aprendizagem Ativa em Administração: um Estudo da Aprendizagem Baseada em Problemas (PBL) na Graduação". Revista Pretexto, vol. 15, n. NE, 2014, pp. 29-47.

SPRONKEN-SMITH, R. \& HARLAND, T. "Learning to Teach with Problem-Based Learning". Active Learning in Higher Education, vol. 10, n. 2, 2009, pp. 138-153.

ZABIT, M. N. M. "Problem-Based Learning on Students' Critical Thinking Skills in Teaching Business Education in Malaysia: a Literature Review". American Journal of Business Education, vol. 3, n. 6, 2010, pp. 19-32.

Publicado em 12/06/2018. 